\title{
A Study on Shrinkage and Warpage of Rotational Moulded Polyethylene
}

\author{
L. Costa; M.C. Cramez, A.J. Pontes \\ IPC - Institute for Polymers and Composites, University of Minho, \\ I3N, Institute of Nanostructures, Nanomodelling and Nanofabrication \\ 4800-058 Guimarães, Portugal \\ e-mail: pontes@dep.uminho.pt
}

Keywords: Rotational moulding; Polyethylene; Shrinkage; Warpage.

\begin{abstract}
Warpage and poor dimensional stability of rotomoulded products are two of the main obstacles to the use of this technique in the production of engineering parts. The knowledge of the effect of the processing conditions on the shrinkage of rotomoulded parts will allow overcoming some of the restrictions of this process.

In the present work the influence of the processing conditions on the development of shrinkage and warpage of rotomoulded parts was studied. The moulding of the parts was performed using a rotational moulding machine build at the University of Minho. The shrinkage and the warpage of the moulded parts were assessed using 3D MMC (3D measuring Machine Control) equipment, and understanding the microstructural development.
\end{abstract}

\section{Introduction}

All materials used in the manufacture of plastic products shrink. The shrinkage is a decrease in the volume of parts during the cooling phase of the plastic. This is quantified by the difference between the dimensions of the part and the dimensions of the moulding cavity from which it originates. The differentiated shrinkage is the main cause of various types of warpage.

Shrinkage occurs during the cooling phase of polymeric material. In the case of semi-crystalline plastics, the use of a slow cooling allows the crystallization of the material, enhancing the increase in shrinkage. In turn, the fast cooling prevents crystallization and this result in a more amorphous structure and smaller shrinkage.

Another factor affecting the shrinkage, appointed by R.J. Crawford [1], is related to the temperature at which the part separates from the mould wall. An early separation, at high temperature, provides a slow cooling, which is reflected in the effects presented above. On the other hand a later separation increases the cooling rate, reducing the shrinkage.

The large thickness of the parts is also a cause of high shrinkage due to the greater difficulty that exists in cooling all the wall thickness at the same rate. This leads to different degrees of crystallinity, lower in the zone next to the mould (mould surface), rising to the interior of the part (inner surface). An equal shrinkage in all directions reduces the warpage [1].

Warpage is often cited as one of the reasons that limit the success of rotational moulding $[2,3]$. A number of factors have been identified that affect the development of residual stresses and the dimensional stability of moulded parts. Most studies focused on evaluating the effect of the processing conditions on the development of warpage, shrinkage, and residual stresses. Bawiskar et al [4] compared the levels of warpage developed in parts made from different thermoplastics and concluded that the cooling phase was the most important factor.

Warpage can be divided into three types - convex, concave and irregular. During the cooling, the air inside the mould tends to reduce its volume. This phenomenon pulls the inner part, and this will exert a compressive force on the hot plastic part at both ends A and B, Fig. 1. 


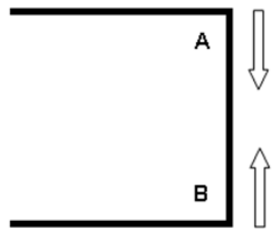

Fig. 1 - Compressive force that the part is subject to while it is hot [3].

When the plastic in the mould is at a high temperature, the stiffness of the part is relatively low. The force applied at both ends of the part will led to the deformation of the surface. The result will be concave and / or irregular warpage.

The time when the fast cooling is applied (cold water spray) is crucial for the development of warpage. If it is applied at a later stage of the cooling, the part is cool enough and with sufficient stiffness to resist the vacuum force that it is being subjected to. In the case of the fast cooling being applied early in the parts, the temperature is still high and the stiffness of the parts low. Thus, the vacuum force prevails over the stiffness of the part, and warpage occurs [3].

Another difficulty is linked to the fact that the mould cooling starts at the outer surface of the part, while the inner surface is still hot. This temperature gradient results in an imbalance of residual stresses that tend to balance out resulting in deformation of the part, in the form of convex warpage. The three types of warpage are shown in Fig. 2.

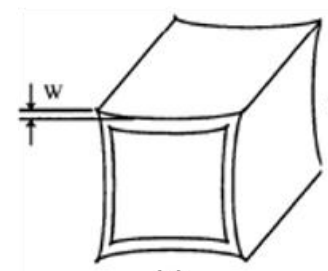

(a)

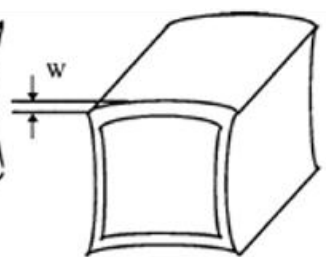

(b)

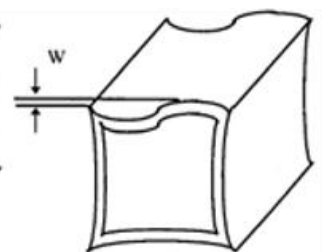

(c)

Fig. 2 - Different types of warpage: (a) concave, (b) convex and (c) irregular [3].

\section{Experimental}

The parts were produced using a rotational moulding machine with a cubic aluminium mould coated with PTFE $(90 \mathrm{~mm} \times 90 \mathrm{~mm} \times 140 \mathrm{~mm}$, thickness $5 \mathrm{~mm})$. The material used was polyethylene, Total Petrochemicals PE M 3583 UV with MFI of $8 \mathrm{~g} / 10 \mathrm{~min}\left(190^{\circ} \mathrm{C} / 2.16 \mathrm{~kg}\right)$. This material is a second generation metallocene medium density polyethylene (mMDPE) with hexene as comonomer. The variables studied were the shot weight - 100, 150 and $200 \mathrm{~g}$, the cooling medium - air and water, and the demoulding temperature -60 and $90{ }^{\circ} \mathrm{C}$. The PIAT (Peak Internal Air Temperature) was kept at $220 \pm 5^{\circ} \mathrm{C}$. Three parts were moulded for each set of conditions.

The microstructure of the parts was characterized using polarized light microscopy and SALS (Small Angle Light Scattering). Although no studies are known in rotational moulding, the SALS technique has been used to assess the size of the spherulites in semicrystalline plastics processed by other methods, such as injection moulding [5].

The density of the parts was measured using a density gradient column.

For all the tests the samples were $15 \mu \mathrm{m}$ thick sections, cut across the thickness of the parts using a microtome.

The dimensions of the moulded parts were measured using a Tesa micro-Hite 3D. The measures were taken both at the fixed and mobile part of the mould/part. A number code was used for measurements across the width of the part and a letter code was used for the measurements correspondent to half the depth of the part, Fig. 3. 


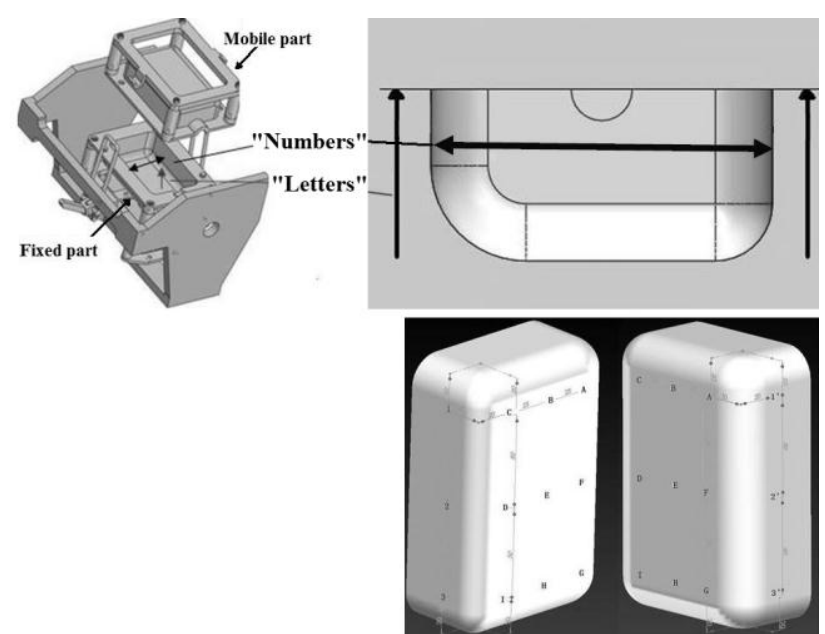

Fig. 3 - Mould and dimensions used for shrinkage assessment.

The shrinkage was defined as the difference between the dimension of the part and the dimension of the mould, at the same point. This value evaluates how much the part deviates from the mould at that point.

The warpage of the parts was qualitatively obtained from the values of shrinkage.

\section{Results and Discussions}

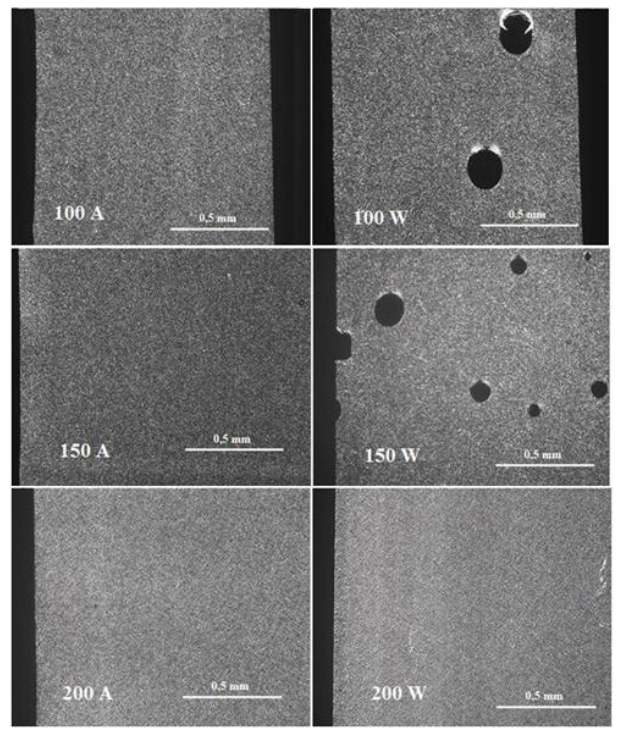

Fig. 4 - Polarized light micrographs of the mouldings (left images air as cooling medium, left imagens water as cooling medium).

From the microstructure results shown in Fig. 4, we concluded that:

A) the number of bubbles increases with the cooling rate, being higher when water is used as cooling medium. This is due to the shorter time available for the diffusion of air out of the molten material;

B) when the cooling rate is higher (done with water), the formation of crystalline nuclei is favoured, resulting in a decrease in the size of the spherulites, Fig.; 


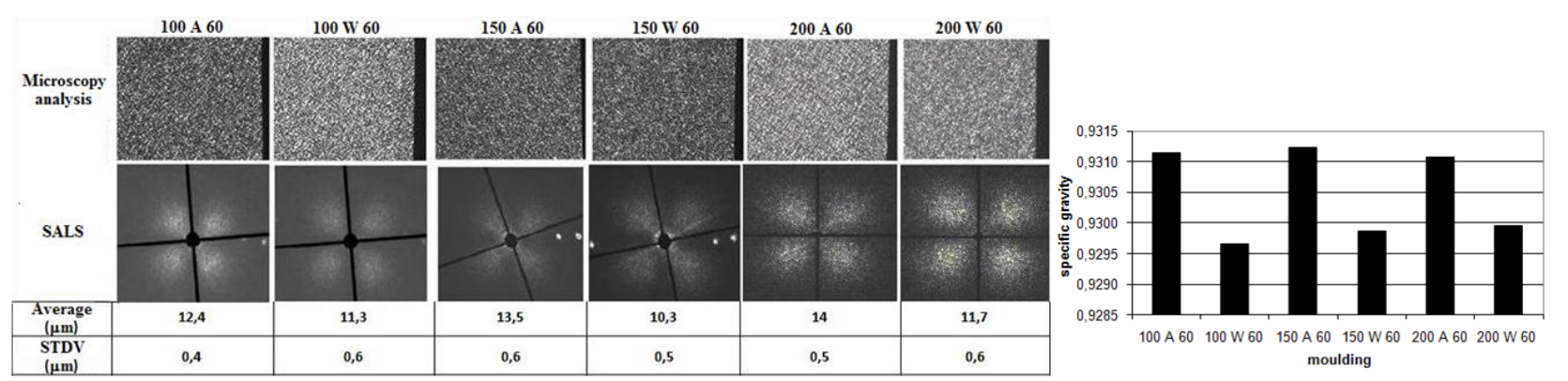

Fig. 5 - SALS, values of the spherulites size and Density results.

C) when the shot weight is increased, the cooling becomes slower and the average diameter of the spherulites increases. This effect is quite clear in the air cooled samples;

D) density decreases in the samples cooled with water, which can be attributed to a decrease in the crystallinity of the samples, due to the faster cooling. A small increase in density with the shot weight was observed in the water cooled samples;

The shrinkage differs if the surface of the part was moulded on the "Fixed" or on the "Mobile" part of the mould. In the "Mobile" part the shrinkage is constant around 1.5\%, Fig. 6 and the part does not show warpage.

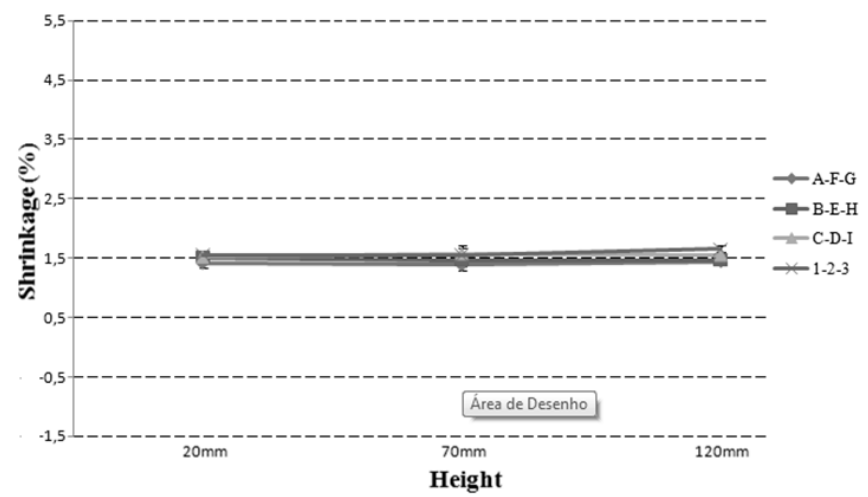

Fig. 6 - Shrinkage along the height of the "Mobile part", condition code 100A60.

In the "Fixed" part the variation on the shrinkage resulted on two types of warpage - concave and convex, Fig. 7.

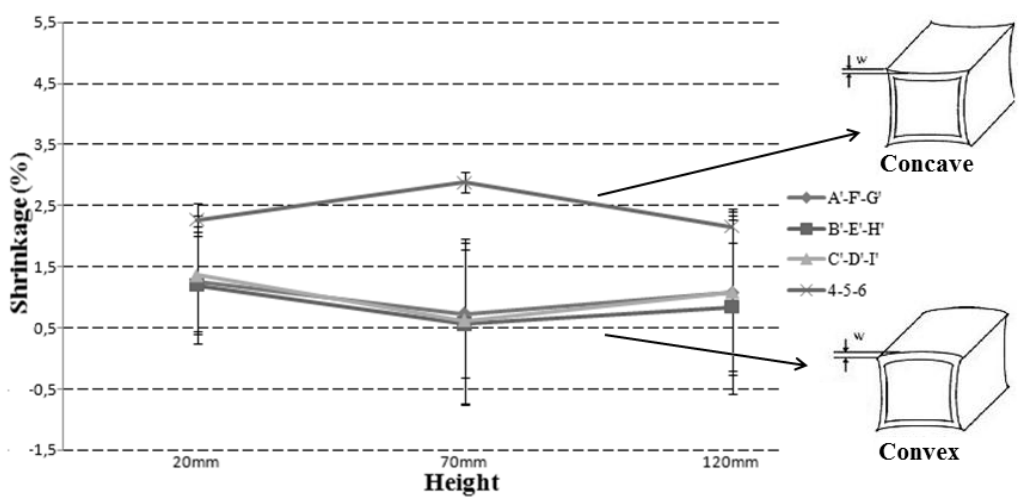

Fig. 7 - Shrinkage along the height of the "Fixed part", condition code 150A60

The explanation for this different behaviour is related to the different metal mass that surrounds each part of the mould, which originates different thermal gradients. As can be seen in Fig. 8, the temperature difference reaches about $15^{\circ} \mathrm{C}$ during the heating of the material and about $5^{\circ} \mathrm{C}$ during the crystallization. 


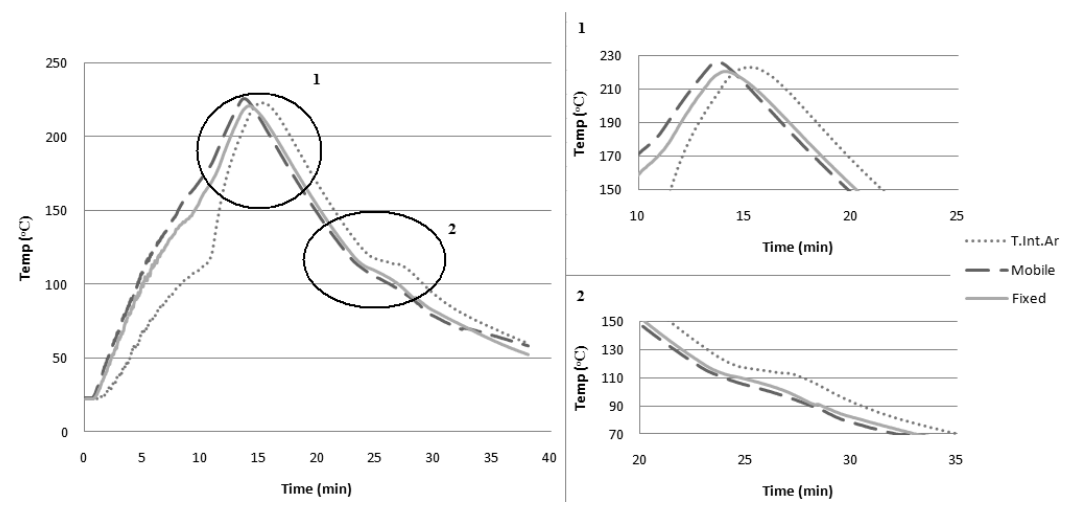

Fig. 8 - Temperature in the two halves of the mould during a moulding, condition code 200A60.

The results obtained across the width of the part (coded with numbers) showed very different values in the mobile and in the fixed part. One explanation for these results can be the one proposed by Crawford [1]. When the parting line does not guarantee the tightness of the mould, the outside air pressure pushes the wall of the part into the mould. As the "Fixed part" is relatively warmer, yields more easily to the pressure and the dimensional variations are more pronounced in this area.

The increase in the shot weight resulted in a decrease in the shrinkage. This means that the increase in the stiffness of the part was more important than the increase in crystallinity. The increase in stiffness prevented the part from deviate from the mould dimensions. The warpage pattern was similar for the three values of the shot weight used, Fig. 9.
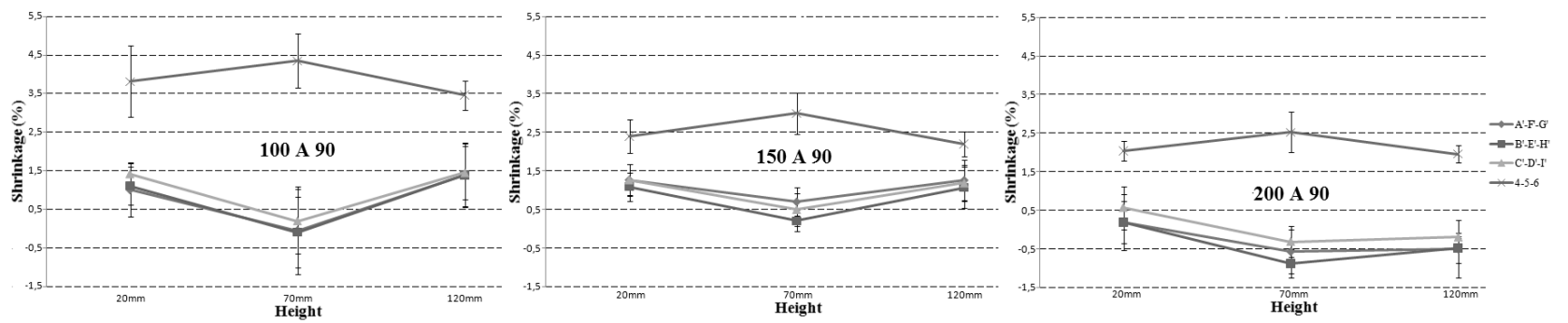

Fig. 9 - Shrinkage along the height of the "Fixed part", keeping A 90.

The water cooling attenuates the stiffness increase observed previously, Fig. 0, because the part suffers less crystallization and as a result the value of shrinkage remains almost constant.
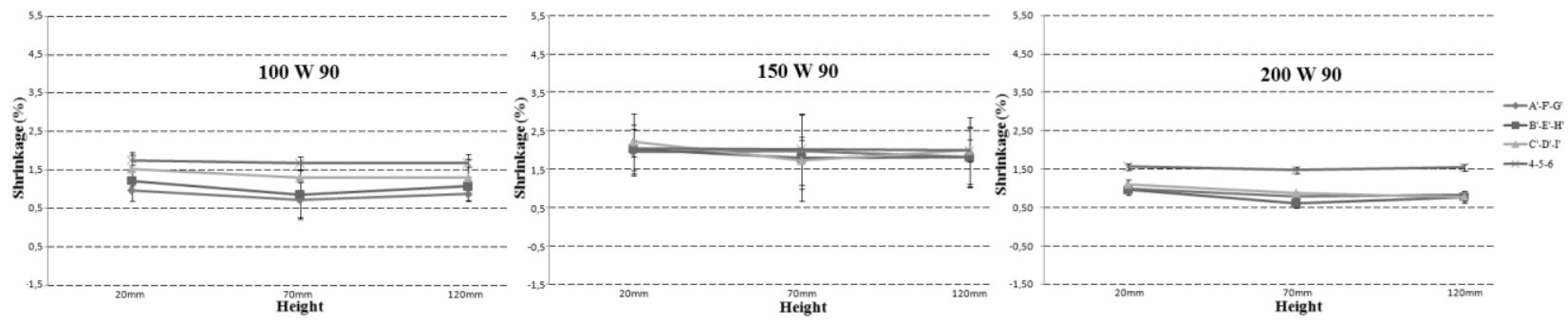

Fig. 10 - Shrinkage along the height of the "Fixed part", keeping W 90.

As noted above, when air-cooling is used, large fluctuations in the values of the shrinkage (more warpage) on the fixed part are observed. The use of water cooling reduces the variability of the results along the parts as well as the values of the shrinkage (less Warpage), in accordance with the results reported by R. J. Crawford [1].

The results for a demoulding temperature of $90^{\circ} \mathrm{C}$ are presented in Fig. 10. The same tendency was observed when the mouldings were removed at $60^{\circ} \mathrm{C}$, as can be seen in Fig. 31. 


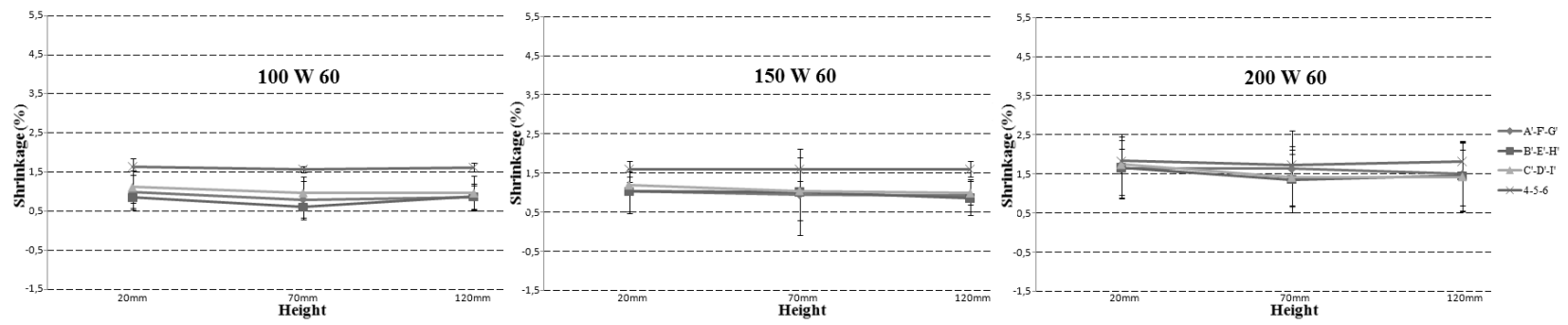

Fig. 3 - Shrinkage along the height of the "Fixed part", keeping W 60.

The removal of the part at a high temperature increases the dimensional variations resulting in more warpage. This is because the part is removed at a temperature high enough for the material to continue crystallizing and shrinking.

During the cooling, while the part is inside the mould, there is adhesion between the two until a certain stage. At this stage the mould acts as a constraint to shrinkage. When the part is removed it acquires more freedom to shrink.

\section{Conclusions}

The results showed that the cooling stage of the cycle is of critical importance to the dimensional stability of the parts. The causes for warpage were also identified. One of the causes is the fact that only the external surface of the plastic part is in contact with the metal mould, developing thermal gradients along the thickness of the part. This is aggravated by the fact that the thicknesses generally used in rotational moulding are high to guarantee an adequate mechanical performance of polyethylene parts.

It was verified that for the highest thickness, the increase in stiffness can hinder the occurrence of shrinkage and warpage in the moulded parts. The effect of the cooling medium (water or air) and demoulding temperature was also analysed. Water cooling led to more homogeneous shrinkage across the parts, and consequently to a decrease in warpage. A higher demoulding temperature resulted in more warpage, due to an increase in the variation of the shrinkage.

The shrinkage of the moulded parts was affected by the support system of the mould as it was found that the "fixed part", surrounded by a large mass of metal, undergoes different thermal gradients in relation to the "mobile part", which caused differences in the shrinkage and warpage results.

In the warpage study, a significant difference was observed between the behaviour of the "mobile" and the "fixed part". There is a greater tendency for the occurrence of warpage at the "fixed part".

\section{Acknowledgments}

The authors acknowledge the financial support given by the FP7 European project - Rotoflex.

\section{References}

[1] Crawford R. J. - Shrinkage, Warpage and Residual Stresses in Rotational Moulding -Causes and Cures. Lecture, Rotational Moulding Seminar, Universidade do Minho, Guimarães, 9 - 10 September 2004.

[2] $\mathrm{Xu} \mathrm{H.;} \mathrm{Bellehumeur} \mathrm{C.T.} \mathrm{-} \mathrm{Thermal} \mathrm{Residual} \mathrm{Stress} \mathrm{Development} \mathrm{for} \mathrm{Semi-Crystalline}$ Polymers in Rotational Molding. Polym. Eng. Sci. Feb, 2008.

[3] Liu S. J.; Ho C.Y. - An Experimental Investigation of the Warpages in Rotationally Molded Parts. Journal of Reinforced Plastics and Composites, Vol. 19, No. 12, 992-1002 (2000).

[4] Bawiskar S.; White J. L. - Comparative Study of Warpage, Global Shrinkage, Residual Stresses, And Mechanical Behavior of Rotationally Molded Parts Produced From Different Polymers. Polym. Eng. Sci., 34 (1994) p.815.

[5] Viana J. C.; Cunha A. M.; Billon N. - The effect of the skin thickness and spherulites size on the mechanical properties of injection mouldings. Journal of Materials Science 36 (2001). 